\title{
De la vaccination
}

\section{Eberhard Wolff}

Prof. Dr rer. soc., rédacteur culture, histoire, société

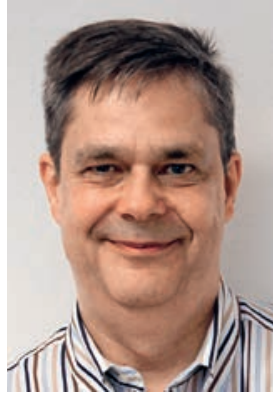

Ces derniers mois, les médias suisses et internationaux ont une fois de plus beaucoup parlé de la vaccination, notamment en raison de récents cas de rougeole. Le tumulte médiatique sur ce thème n'est pas nouveau. Il suffit de jeter un coup d'œil aux anciens numéros du BMS pour s'en convaincre. Ces dernières années, on y retrouve de vastes - et le qualificatif ne se rapporte pas seulement à leur ampleur - débats sur les recommandations de vaccination de la Fondation pour la protection des consommateurs, la campagne pour l'éradication de la rougeole en Suisse d'ici 2015 ou les articles pertinents de la loi fédérale révisée sur les épidémies.

En fait, la vaccination est un sujet qui divise depuis plus de deux siècles, lorsque les premiers vaccins ont été introduits. Pourquoi? A mon sens, cela s'explique notamment par le fait que la vaccination se situe à l'intersection de convictions fondamentales et de systèmes de pensée très différents, qui s'y entrechoquent avec une brutalité que l'on rencontre rarement ailleurs en médecine. Cela transparaît aussi dans la charge émotionnelle et l'intensité des débats sur les questions essentielles de médecine correspondantes. Pour le comprendre, mieux vaut laisser de côté la question de savoir qui a «le plus raison».

Les vaccins étaient et restent une sorte d'essence de ce qui est censé caractériser la médecine moderne, rationnelle, surtout depuis le milieu du XIX ${ }^{\mathrm{e}}$ siècle: agir efficacement, de manière systématique et contrôlée, contre les maladies des individus et des communautés, de préférence avant même qu'elles se déclarent. Longtemps, la prévention et finalement l'éradication de la variole ont fait la fierté de toute une profession. L'élimination des maladies a toujours été une grande utopie de la médecine. Le scepticisme ou la critique de cet idéal sont, entre bien d'autres choses, considérés comme des doutes sur l'image fondamentale que cette médecine a d'elle-même.

Mais les vaccins sont aussi un exemple éclatant de médecine interventionniste, plus particulièrement d'une intervention sur le corps humain, d'autant plus marquante qu'elle franchit concrètement les limites de la peau humaine. Hier avec des incisions, aujourd'hui avec des injections. En ce sens, les vaccins cristallisent également des conceptions différentes de l'importance que le corps humain, la «nature» ou le «naturel» doivent revêtir en médecine, de la manière dont cela doit se traduire et du caractère invasif, «artificiel» ou «synthétique» admissible ou possible pour la vaccination.

Différentes notions de risque se heurtent aussi dans les débats. Quel poids accorde-t-on aux risques d'une maladie ou de sa prévention et comment les met-on en balance? S'agit-il du risque pour un individu ou pour un groupe? Est-il considéré d'un point de vue extérieur ou de celui de l'individu lui-même? La notion abstraite de risque est en outre étroitement liée au sentiment concret de peur: le débat se charge très vite d'émotions. Les avis divergents sur les vaccins nous confrontent par ailleurs à d'autres interrogations: quelle pluralité voulons-nous pour notre système de santé et où doivent en être les limites?

Les vaccinations ne concernent pas seulement la médecine. Elles soulèvent (au-delà du thème de la vaccination obligatoire) toujours la question - essentielle pour notre coexistence - du rôle de l'individu par rapport à la société dans son ensemble. Quand la collectivité a-t-elle le droit, le devoir ou l'obligation d'empiéter sur l'autonomie de l'individu et quand est-il préférable qu'elle s'abstienne? Dans quelle mesure, la valeur "santé» est-elle absolue comparée à d'autres comme la liberté individuelle? Qu'est-ce qui prime entre la logique de la santé et les libertés civiles?

Et il ne s'agit là que de l'énumération de quelques mots clés. Chacun d'eux peut déclencher des débats passionnés. Associés, ils s'influencent comme les éléments d'un mobile.

La vaccination était et reste un sujet médical brûlant, parce qu'elle cristallise des questions fondamentales et imbriquées de la médecine, que chacun voit différemment: utopies et dystopies, efficacité et pluralité, sécurité et risque, intervention et naturel, individu et société, liberté et responsabilité. Le récent tapage médiatique sur la vaccination ne sera pas le dernier. 